\title{
Viral Forecasting, Pathogen Cataloging, and Disease Ecosystem Mapping: Measuring Returns on Investments
}

\author{
Jeanne Fair and Joseph Fair
}

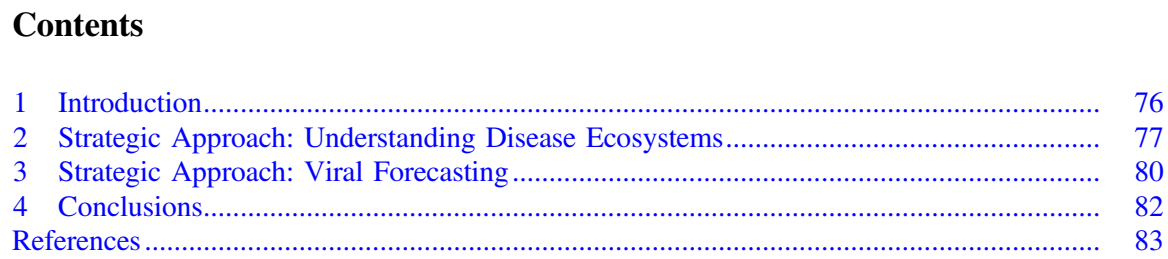

\begin{abstract}
Infectious disease emergence into humans from animals or the environment occurs primarily due to genetic changes in the microbe through mutation or re-assortment making it either more transmissible or virulent or through a change in the disease "ecosystem". Research into infectious disease emergence can be grouped into different strategic approaches. One strategic approach is to study a specific or model disease system to understand the ecology of an infectious disease and how is transmitted and propagated through the environment and different hosts and then extrapolate that disease system knowledge to related pathogens. The other strategic approach follows the genomics and phylogenetics - tracking how pathogens are evolving and changing at the amino acid level. Here we argue that for understanding complex zoonotic diseases and for the purposes of preventing emergence and re-emergence into humans, that the Return on Investment be considered for the best research strategy.
\end{abstract}

\footnotetext{
J. Fair

Biosecurity \& Public Health, Los Alamos National Laboratory, Mailstop M888, Los Alamos, NM, USA

J. Fair (ه)

The Scowcroft Institute of International Affairs, the Bush School of Government and Public Service, Texas A\&M University, College Station, TX, USA

e-mail: curefinder@icloud.com
}

Current Topics in Microbiology and Immunology (2019) 424: 75-83

https://doi.org/10.1007/82_2019_179

(C) Springer Nature Switzerland AG 2019

Published Online: 16 October 2019 


\section{Introduction}

Advances in molecular biology and genomics are revolutionizing our understanding of infectious diseases and the mechanisms and pathways by which they emerge into human, animal, and plant species and occasionally result in catastrophic events that range in size from an affected household, village or herd, to a global pandemic or large-scale die-off of livestock. Catastrophic biological events such as a lethal pandemic can and do result in a massive loss of human and animal lives and billions (potentially trillions) of dollars in affected nations and regions. Even though infectious diseases have killed more humans and animals throughout history than all wars in human history combined, pandemic threats are rarely among the top concerns of nation-states, which usually focus on nuclear and to a much lesser extent, chemical weapons, and other weapons of mass destruction that include weaponized biological organisms. While our ability to detect these pathogens is ever closer to real time, the increased species and environmental interactions that drive pathogen evolution are occurring much faster than our global patchwork of infectious disease surveillance efforts can detect them. By considering emerging pathogens less of a threat to national security than those poised by relatively complex human-engineered weapons systems (like nuclear weapons), we risk nothing less than our ability to survive as viable and sovereign nation-states.

Infectious disease emergence into humans from animals or the environment occurs primarily for two reasons: The first is due to genetic changes in the microbe through mutation or re-assortment, making it either more transmissible or virulent. The second is through a change in the disease "ecosystem." The disease ecosystem refers to the environmental and species interactions that either directly or indirectly lead to the emergence of infectious diseases in one or several species. Examples of this include the zoonotic transfer of pathogens through bushmeat consumption, or by human migrations into previously undeveloped areas, leading to increased interactions with and consumption of wildlife that can and do often harbor pathogens harmful to humans or livestock. The One Health Initiative is a multidisciplinary approach to study the interaction between humans, pathogens, livestock, wildlife, and the environment, and how they can ultimately lead to the emergence of disease.

Research into infectious disease emergence can generally fall within two different strategic approaches. The first strategic approach is to study a specific or model disease system to try and understand the ecology of the disease and how is transmitted and propagated through the environment and different hosts than to extrapolate that disease system knowledge to related pathogens. This type of research is usually designed to be done at one (or a few locations), longitudinally, collecting a plethora of data types in an attempt to understand as much as possible about the disease ecosystem and what leads to the emergence in humans or animals. It is a "deep dive" to understand how humans, wildlife, weather, agricultural animals, plants or anything else might impact or play a role in the continued spread of a disease or ecology of its transmission and movement. It is important to note that phylogenetics of genetic changes in a pathogen will likely play a vital role in this last strategy. The data 
collected for the second strategy can span between the genomic to the geography and climate. The other strategic approach follows the genomics and phylogenetics of pathogens tracking how pathogens are evolving and changing at the amino acid level. The latter is presently the most common line of research, particularly for understanding both seasonal influenza and trying to predict potential pandemic influenza. This strategy is also to look for and catalog new viruses and bacteria in different hosts and environments to understand the overall biodiversity of microbes and pathogenic strains in an effort akin to creating a global genomic database of pathogens and where they originate. The goal is to understand and map pathogens to be more aware of where they might be lurking and display them in the form of heatmaps, with areas of high emergence deemed emerging infectious disease hotspots.

The use of genomic monitoring and pathogen cataloging, rarely leads to the identification of the spillover event or its cause, either at a macro- or micro-level. Of note, however, recent advances in field portable sequencing technologies have led to a more real-time analysis of strain-specific pathogen transmission in humans and animals and have aided in identifying transmission chains and index cases in several outbreaks. In order for us to prevent infectious disease emergence (or re-emergence) into humans, animals, and livestock, it is crucial to know how the pathogen first emerges and transmitted into humans or animals. It is also crucial to understand how the disease may manifest differently depending on the individual host. Not every animal exposed is a host and not every host reacts in the same way, leading to different disease manifestations and outcomes in many cases. If we further understand how a disease is transmitted or the overall ecosystem of the pathogen, we can develop both high- and low-tech interventions to break the chain of transmission. Historically, the most successful interventions in limiting the spread of deadly infectious diseases were developed by observing and developing a keen understanding of the complete lifecycle of the pathogen or its ecosystem. This tenet is particularly true for zoonotic diseases that have emerged or re-emerged into humans. Moreover, most of the aforementioned disease hotspots are in regions of the developing world, where high technology platforms most often fail and cannot be sustained.

\section{Strategic Approach: Understanding Disease Ecosystems}

In April 1993, near Gallup, New Mexico, a young Navajo woman arrived at the Indian Medical Center emergency room with flu-like symptoms and severe shortness of breath. Doctors found the woman's lungs filled with fluid, and she died soon after her arrival. The cause of her death could not be immediately determined, and the case reported to the New Mexico Department of Health. Five days later, her fiancé, was en route to her funeral in Gallup when he suddenly became ill with severe shortness of breath. By the time paramedics brought him to the Indian Medical Center emergency room, he had stopped breathing. Doctors could not revive the young man and also died. It was clear that a deadly infection caused both deaths. However, by what microbe? 
Over the next two weeks, the New Mexico Department of Health Infectious Disease Specialist, Dr. Gary Simpson, sought to find out what killed this young couple. Bringing together epidemiologists, mammologists, environmental scientists, GIS specialists, and climatologists, Dr. Simpson and the team eventually discovered that the outbreak was caused by the Sin Nombre Hantavirus, transmitted by wild small mammals, primarily North American deer mice (Peromyscus maniculatus), through breathing in aerosolized virus particles, by sweeping up dry mice droppings (Pennington et al. 2013). Over the next decade, the team and others would discover that there was not only a seasonality to hantavirus infections, but that infections increased in years following El Nino climate years and the host range virus (Yates et al. 2002; Mills et al. 1999; Hjelle and Yates 2001; Brunt et al. 1995; Calisher et al. 2011).

By developing an understanding of the ecosystem and ecology of hantavirus in the southwest, the team saved human lives. Using the information on the ecology of hantavirus in the environment, public education on the risks of deer mice droppings is now widespread in at-risk National parks in the USA, sensitizing US National Park patrons to the risk of the disease and how to safeguard themselves. Since the first cases of Sin Nombre hantavirus, researchers, primarily at the University of New Mexico, created a science-based knowledge and understanding of hantavirus in this wildlife-human interface (Calisher et al. 2011). Hantaviruses would never be able to be eradicated due to its zoonotic reservoir (a common rodent); thus, like with most zoonotic infectious diseases, we must learn how to avoid infection through behavior.

Another example of understanding an emerging infectious disease ecosystem is Lyme disease in eastern North America. Lyme disease, caused by the bacterium, Borrelia burgdorferi, continues to infect thousands of people per year with no decrease in sight. Host susceptibility or effects in humans are variable with many cases resulting in complete debilitation to the patient. In an eloquent series of studies, researchers have discovered that the bacteria transmitted by deer ticks (Ixodes scapularis) and that deer themselves are only indirectly involved. Again, a multidisciplinary team of researchers used the Understanding Disease Ecosystems approach to untangle the complicated story of an exotic grass, small mammal biodiversity and how the urban-wildlife interface has led to an increase in Lyme disease over the last few decades (Ostfeld et al. 2018; Hersh et al. 2014; Ostfeld and Keesing 2012; Keesing et al. 2009; Levi et al. 2012).

Thanks to public health messaging, few people in eastern North American today are unfamiliar with Lyme disease and its link to ticks in the summer months. Most people are aware that they should look for the bullseye ring around a tick bite. Again, through understanding the disease system, efforts could be focused to educate the public on the potential transmission pathways and how to avoid infection, rather long-term, expensive, and complex solutions like a vaccine. Additional lessons on how the reduction of biodiversity can lead to the propagation of pathogens (Keesing and Ostfeld 2015) were learned from this experience.

These two disease ecosystem examples demonstrate that just identifying the microbe without understanding the complexity of the disease system would not have led to understanding the transmission route and possible mitigations for 
curtailing outbreaks. There are currently 1700 infectious diseases and parasites that infect humans with over $75 \%$ of them being zoonotic (Blancou et al. 2005), and outbreaks continue to increase (Smith et al. 2014). While it would be cost and time prohibitive to understand each zoonotic disease system, much can be extrapolated to other diseases by obtaining an in-depth understanding of one model disease ecosystem. The role of changing environments, increased human-wildlife interaction, increased vector ranges that accompany changes in climate, and the evolution of microbes and host response are vital to understanding how we can mitigate the spread of lethal epidemics/pandemics. As the interactions between humans, wildlife, and the environment continue to increase in frequency and scale, understanding the ecosystem of a pathogen is the fundamental objective of the One Health methodology.

Evolutionary biology, synecology, and autecology techniques and methods, cutting-edge wildlife mark-recapture techniques, survival analysis, spatial GIS analysis, cost-effective and accurate sequencing and bioinformatic analysis, epidemiological modeling, remote sensing and climate modeling can be coupled with updated and sophisticated statistical analysis and experimental designs to test hypotheses in the field in order to understand a disease ecosystem and what leads to emergence and spillover. A multidisciplinary approach, like that used to discover the cause of Four Corners hantavirus outbreak, can lead to transformative science (Pennington et al. 2013). While this is not a small, cheap, or easy endeavor, this type of research can lead to a high return on investment (ROI). Much-like complex mathematical models coupled with data feeds are used to predict the value and returns on stocks and commodities, and disease surveillance programs can and should be categorized by their individual returns on investments or ROIs.

Conducting fieldwork can be risky when compared to controlled laboratory work, because there is always something to learn in the field as it is a dynamic ecosystem, instead of a controlled artificial environment in the laboratory. A negative result in field biology is, in fact, learning about the environment and ecosystem and identifying the patterns and interactions that lead to the emergence of pathogenic organisms. For over 70 years, wildlife science has sought to understand ecological systems in order to better manage and maintain the health of those systems. This approach has evolved over the decades from extradition of species to protecting species and better managing game populations. Zoonotic disease control is now a part of that management of many species, and while vaccines and antibiotics have been revolutionary in reducing global mortality in both humans and animals, the subsequent increases in antibiotic resistance are a considered a significant threat to global health security.

Both hantavirus and Lyme disease are examples by which understanding the ecology of an infectious disease system directly led to wide-scale and highly effective, cost-efficient mitigations that are in place until today. While both pathogens continue to infect humans and animals, their overall impact was dramatically decreased by applying a one health investigative strategy. It was through deep-dive investigations to understand these disease ecosystems, and, specifically, how weather, plant life, host heterogeneity, biodiversity, drought, human behavior, 
vectors, wildlife, and reservoirs' species all can play a role in the emergence of deadly pathogens into humans and animals. Both of these examples of understanding the ecology of an infectious disease system have led to public education, policy, and management practices changes, leading to an overall and dramatic reduction of infections. The funds to support the researchers involved in this work have undoubtedly shown a healthy return of investment (ROI).

\section{Strategic Approach: Viral Forecasting}

The majority of US foreign aid designated to fight the emergence of novel pandemics over the past decade has been devoted to "viral forecasting" in regions known to have a high incidence of zoonotic disease transmissions or spillover events. Such geographic locations have been identified as emerging infectious disease hotspots (Jones et al. 2008). Commonly displayed in the form of heat maps, such infectious disease hotspot maps have indicated for many years that West Africa was consistently at higher risk than average for a large-scale epidemic. However, the West African Ebola outbreak of 2014 proved that subjective/ qualitative knowledge of an area prone to disease is not sufficient to prevent an epidemic. Interventions to protect humans from the emergence of viral diseases in hotspots are possible but only with early and high-resolution visibility into the ecology of viral emergence along with a new set of tools to suppress pathogens before they result in epidemics. The USAID PREDICT Program collected samples from four key animal taxa (and others) thought or suspected to carry those pathogens known to spillover in humans. They equipped many disease hotspot nations with US University-developed plasmid-based standard PCR assays for different viral pathogens that are used to broadly screen animal samples collected by USAID-funded surveillance activities in those countries. Samples are collected, nucleic acids extracted, and collection and any available metadata entered into databases. Samples are shipped back to the USA and sequenced at US academic partner laboratories, all of whom use different techniques and bioinformatics approaches. This process allows for diagnosis of disease but cannot distinguish between viral quasi-species as of yet and relies upon sequencing cores based in the USA and the months of protocols and procedures that require to facilitate full genomic analysis. Individual institutions and groups implement the PREDICT program, and countries are divided by the institution, rather than by scientific focus.

Over the past several decades, the USA and the world have invested hundreds of millions of dollars on these different strategies for researching infectious disease emergence. While each type can play an essential role in reducing the threat of infectious diseases, the two types may have significantly different ROIs. The first strategic approach of research into understanding the ecology of infectious disease has led to critical insights on disease systems that were used to develop biosurveillance strategies and mitigations. While the research may have focused on one location, the results can be extrapolated to other locations and potentially other 
diseases. The second strategy of cataloging pathogens and tracking the phylogenetics of pathogens continues to be critical for tracking seasonal and newly emerging diseases to understand genetics changes that may impact virulence and transmission. The second strategy has collected hundreds of thousands, if not millions, of samples around the globe and has led to some discoveries such as finding a new Ebola virus in bats.

The strategy of disease system ecology can be funded by different agencies (an interagency approach using a combination of aid, scientific research, and privately funded initiatives) and on a project by project basis, focusing the diseases which we know pose the most significant risk to humans and livestock and wildlife. In 2011, the National Institutes of Health and National Science Foundations joined forces to create a specific research call entitled Ecology and Evolution of Infectious Diseases (EEID). While a successful program from the start, this program has become increasingly more competitive over the years, with more and more applicants and fewer dollars allocated to each study. While there is no data to support the assertion, researchers have reported being told for any project that seemed to investigate the ecology of infectious disease, the investigators should submit to EEID instead. Figure Pie chart of potential funding for each category

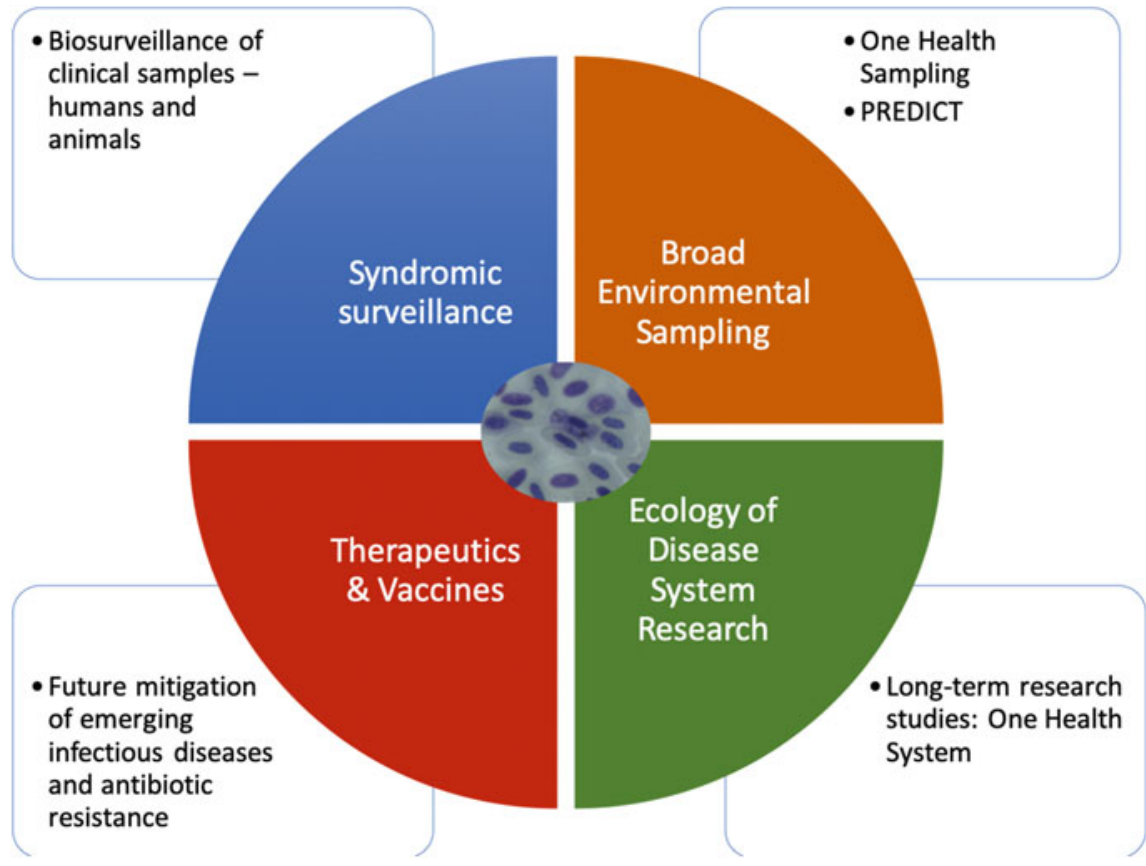




\section{Conclusions}

Here, we argue that by developing a keen understanding of the complex interactions that lead to zoonotic disease, spillovers are a more proven method to prevent emergence and re-emergence of pathogenic infectious diseases in humans that the ROI for disease ecology system is higher than the aforementioned viral forecasting and pathogen cataloging strategies. That is not to say that these programs do not offer great value to microbiology and science; they without question do. However, to categorize them as a means to either predict or respond to lethal infectious epidemics is somewhat misleading. In more than ten years of dedicated programming and hundreds of millions of dollars, no lethal epidemics have been predicted or prevented. The advances in genomics and sequencing technologies are increasingly being incorporated into outbreak response and have provided invaluable insights into disease transmission chains. These technologies are predicted to become even more robust and standardized in the coming years and will continue to play essential roles in molecular epidemiology and disease transmission chain identification. Viral and pathogen cataloging is essential to science, but if our aim is to protect humans, animals, and livestock, this approach will have minimal return on investment and should arguably remain in the category of "museum" science where we continue to catalog and under the biodiversity of life on this planet, both in the present and those that existed before the emergence of humankind.

History has taught us that certain types of diseases and syndromes lend themselves to emerging into highly lethal and catastrophic epidemics and pandemics. Some are seasonal and ever-present, like influenza and Lassa fever, while others are still at the stage of individual spillover events, like SARS and MERS. Still, other diseases that were previously considered no longer threats to humankind are re-emerging as threats to human lives due to the societal shifts toward anti-vaccination attitudes and behaviors. By studying model disease ecosystems in each of the categories that we know can and have killed millions in the past, we can prepare ourselves to respond to both known and novel threats that will continue to emerge as increased pressures on the environment change the earth's ecosystem dynamics. If we have learned anything from history, it is that we need to be nimble, flexible, and ready to respond to these diseases as they emerge. In the future, it may indeed be possible to predict disease emergence using a combination of technology, social awareness, and continual surveillance for pathogens and other epidemic indicators. However, at the time of this writing, more than a decade of disease prediction and pathogen cataloging efforts at enormous costs to the public has had a measurably low return on investments, as evidenced in the major epidemics that have occurred during that time. To safeguard our future, we have to understand the ecosystems of lethal pathogens, and from that knowledge, be able to extrapolate and adapt as new threats emerge. 


\section{References}

Blancou J, Chomel BB, Belotto A, Meslin FX (2005) Emerging or re-emerging bacterial zoonoses: factors of emergence, surveillance and control. Vet Res 36:507-522

Brunt JW, Parmenter RR, Yates TL, Ernest SM, Vigil R (1995) Predicting the hot zone: Rodent population dynamics and the Hantavirus epidemic in the Southwest. Bull Ecol Soc Am 76:33

Calisher CH, Mills JN, Root JJ, Doty JB, Beaty BJ (2011) The relative abundance of deer mice with antibody to Sin Nombre Virus corresponds to the occurrence of Hantavirus Pulmonary Syndrome in nearby humans. Vector-borne and Zoonotic Dis. 11:577-582

Hersh MH, Ostfeld RS, McHenry DJ, Tibbetts M, Brunner JL, Killilea ME, LoGiudice K, Schmidt KA, Keesing F (2014) Co-infection of blacklegged ticks with Babesia microti and Borrelia burgdorferi is higher than expected and acquired from small mammal hosts. PLoS ONE 9:e99348

Hjelle B, Yates T (2001) Modeling hantavirus maintenance and transmission in rodent communities. In: Current topics in microbiology and immunology. Hantaviruses, vol 256, pp 25677-25690

Jones KE, Patel NG, Levy MA, Storeygard A, Balk D, Gittleman JL, Daszak P (2008) Global trends in emerging infectious diseases. Nature 21;451(7181):990-993.

Keesing F, Brunner J, Duerr S, Killilea M, LoGiudice K, Schmidt K, Vuong H, Ostfeld RS (2009) Hosts as ecological traps for the vector of Lyme disease. Proc R Soc Biol Sci 276:3911-3919

Keesing F, Ostfeld RS (2015) Is biodiversity good for your health? Science 349:235

Levi T, Kilpatrick M, Mangel M, Wilmers CC (2012) Deer, predators, and the emergence of Lyme disease. Proc Natl Acad Sci U S A 109(27):10942-10947

Mills JN, Yates TL, Ksiazek TG, Peters CJ, Childs JE (1999) Long-term studies of hantavirus reservoir populations in the southwestern United States: rationale, potential, and methods. Emerg Infect Dis 5:95-101

Ostfeld RS, Brisson D, Oggenfuss K, Devine J, Levy MZ, Keesing F (2018) Effects of a zoonotic pathogen, Borrelia burgdorferi, on the behavior of a key reservoir host. Ecol Evol 8:4074-4083

Ostfeld RS, Keesing F (2012) Effects of host diversity on infectious disease. Ann Rev Ecol Evol Systemat 43:157-182

Pennington DD, Simpson GL, McConnell MS, Fair JM, Baker RJ (2013) Transdisciplinary research, transformative learning, and transformative science. Bioscience 63:564-573

Smith KF, Goldberg M, Rosenthal S, Carlson L, Chen J, Chen C, Ramachandran S (2014) Global rise in human infectious disease outbreaks. J R Soc Interface 11:20140950

Yates TL, Mills JN, Parmenter CA, Ksiazek TG, Parmenter RR, Vande Castle JR, Calisher CH, Nichol ST, Abbott KD, Young JC, Morrison ML et al (2002) The ecology and evolutionary history of an emergent disease: hantavirus pulmonary syndrome. Bioscience 52:989-998 